OPEN ACCESS

Edited by:

Francesca Chiodi,

Karolinska Institutet (KI), Sweden

Reviewed by:

Ruth M. Ruprecht,

University of Louisiana at Lafayette,

United States

Jorma Hinkula,

Linköping University, Sweden

${ }^{*}$ Correspondence:

Morgane Bomsel

morgane.bomse/@inserm.fr

Specialty section:

This article was submitted to

Viral Immunology,

a section of the journal

Frontiers in Immunology

Received: 09 October 2020 Accepted: 01 December 2020

Published: 03 February 2021

Citation:

Xu L, Tudor D and Bomsel M (2021) The Protective HIV-1 Envelope gp41 Antigen P1 Acts as a Mucosal Adjuvant Stimulating the Innate Immunity.

Front. Immunol. 11:599278. doi: 10.3389/fimmu.2020.599278

\section{The Protective HIV-1 Envelope gp41 Antigen P1 Acts as a Mucosal Adjuvant Stimulating the Innate Immunity}

\author{
Lin $X u^{1,2,3}$, Daniela Tudor ${ }^{1,2,3}$ and Morgane Bomsel ${ }^{1,2,3 *}$ \\ ${ }^{1}$ Laboratory of Mucosal Entry of HIV-1 and Mucosal Immunity, Department of Infection, Immunity and Inflammation, Cochin \\ Institute, CNRS UMR 8104, Paris, France, 2 INSERM U1016, Paris, France, ${ }^{3}$ Université de Paris, Paris, France
}

Mucosal nasal vaccine development, although ideal to protect from pathogens invading mucosally, is limited by the lack of specific adjuvant. We recently used P1, a conserved region of HIV-1 gp41-envelope glycoprotein, as efficient antigen in a prophylactic HIV-1 mucosal vaccine applied nasally. Herein, P1 immunomodulation properties were assessed on human nasal mucosal models by measuring induction of cytokine and chemokine production, intracellular signaling pathways, mucosal dendritic cell (DC) activation, and $\mathrm{T}$ cell proliferation. P1 adjuvant properties were evaluated by quantification of antigen-specific B cell responses against a model antigen in an in vitro immunization model. We now demonstrated that $\mathrm{P} 1$ has additional immunological properties. P1 initiates immune responses by inducing nasal epithelial cells to secrete the Th2-cytokine thymic stromal lymphopoietin (TSLP), a described mucosal adjuvant. Secreted TSLP activates, in turn, intracellular calcium flux and PAR-2-associated NFAT signaling pathway regulated by microRNA-4485. Thereafter, P1 induces mucosal dendritic cell maturation, secretion of TSLP in a TSLP-receptor (R)-dependent autocrine loop, but also IL-6, IL-10, IL-8, CCL20, CCL22, and MMP-9, and proliferation of CD4+ T cells. Finally, $\mathrm{P} 1$ acts as an adjuvant to stimulate antigen-specific B cell responses in vitro. Overall, P1 is a multi-functional domain with various immuno-modulatory properties. In addition to being a protective vaccine antigen for HIV prevention, P1 acts as adjuvant for other mucosal vaccines able to stimulate humoral and cellular antigen-specific responses.

Keywords: adjuvant, intra-nasal vaccination route, IgA, mucosa, Th2-cytokine thymic stromal lymphopoietin, HIV-1 gp41, P1, microRNA-4485

\section{INTRODUCTION}

Although most human pathogens initiate infection at mucosal sites, only a few licensed mucosal vaccines have been established so far (1). Intranasal immunization, by inducing an antigen-specific immunity in both the mucosal and systemic compartments and by being applied in a atraumatic manner following pulverization, is currently considered as an ideal strategy for prevention against pathogens invading mucosa (2-4). Of note, the early side effects attributed to nasal immunization, 
including facial nerve paralysis, are no longer a concern since they are attributed to the specific ADP-ribosylating toxin-based adjuvant used in these studies rather than to the nasal immunization route (4-6). Mucosal immunization is highly compartmentalized with unique pathways linking the inductive and effector sites $(2,3)$. In particular, nasal vaccination elicits antigen-specific antibody responses in genital tracts (7) and would be therefore beneficial to prevent transmission of sexually transmitted pathogens. Nevertheless, very limited efforts have been made to understand the mechanisms by which nasal vaccines and dedicated adjuvants activate the local nasal innate and adaptive immunity as a first step to establish an effective vaccination.

P1 is a conserved 35 amino acid peptide covering the Membrane Proximal External Region (MPER) of HIV-1 envelope subunit gp41 $(8,9)$. The MPER is a major target of broadly neutralizing antibodies and thus obviously a very interesting target for an $\mathrm{HIV}-1$ vaccine. In addition, P1 mediates HIV-1 mucosal transcytosis, a principal mucosal entry pathway for HIV-1, by interacting with Galactosyl Ceramide (GalCer), the mucosal receptor of HIV-1 (9-11). Accordingly, we have recently evaluated the protective efficacy of a gp41-subunit-virosome vaccine at mucosal sites in nonhuman primates (8). This vaccine that used P1 as antigen linked to virosomes, an adjuvant-free vaccine carrier, was applied twice by the intramuscular route followed by two intranasal applications. In the primate model, full protection after repeated vaginal challenges with simian-human immunodeficiency virus (SHIV) correlated with P1/gp41specific cervicovaginal antibodies, with IgAs blocking transcytosis and IgGs mediating antibody-dependent cellular cytotoxicity (ADCC). In contrast, in protected animals, serum IgGs totally lacked antiviral activities. Furthermore, in a Phase I clinical trial, we found that P1-virosome vaccination induced mucosal P1-specific antibodies with antiviral activities (12). These results highlighted the critical role of mucosal antibodies as the first line of defense against virus entry.

Thymic stromal lymphopoietin (TSLP) is an IL-7-like cytokine considered as a master regulator of the $\mathrm{T}$ helper 2 (Th2) inflammatory responses by priming dendritic cells (DCs), especially mucosal ones $(13,14)$. We and others recently reported that TSLP is secreted by epithelial cells during HIV-1 mucosal transmission following the interaction of the viral envelope with epithelial cells $(15,16)$. In turn, TSLP chemoattract mucosal DCs to the mucosal compartment (16), suggesting that TSLP could modulate the mucosal immune response following mucosal vaccination. Accordingly, in a study using the HIV-1 envelope gp140 as antigen, TSLP acts as a strong mucosal adjuvant in the mouse model (17). TSLP induced a strong humoral response both in serum and at the genital level following intranasal immunization, comparable to the adjuvant effect of cholera toxin (CT) tested in parallel. In addition, a new study reported that all-trans retinoic acid (RA) shows adjuvant activity through TSLP production (18). Furthermore, a recent study showed that TSLP and TSLPreceptor (TSLP-R) were up-regulated in mucosal DCs of mice nasally immunized with pneumococcal surface protein A plus CT (19) and that in TSLP-R knockout mice, the specific IgA response is remarkably reduced. This indicates that TSLP and its receptor are major contributors to the mucosal adjuvant effect of $\mathrm{CT}$ and that TSLP- TSLP-R signaling is critical in IgA elicitation.

In the present study, we investigated whether $\mathrm{P} 1$, in addition to being an antigen, could act as an adjuvant by first exploring its capacity to stimulate epithelial TSLP production. We then evaluated additional immunomodulatory effects of P1 on human nasal mucosal models, including cytokine and chemokine production, intracellular signaling pathways, mucosal DC activation, T cell proliferation, and antigen-specific $\mathrm{B}$ cell responses against a model antigen in vitro. Altogether, we report the immunological mechanisms underlying P1-vaccine and the potential of $\mathrm{P} 1$ as a nasal mucosal adjuvant.

\section{MATERIALS AND METHODS}

\section{Peptides}

Peptide P1 (aa 650-685) is derived from the HIV-1 gp41 envelope subunit. P1 clade B (SQNQQEKNEQELLE LDKWASLWNWFNITNWLWYIK) is derived from the clade B HXB2 isolate; P1 clade A (SQIQQKKNEQDLLALD KWANLWNWFDISNWLWYIR) from the clade A 99UGA07072 isolate, and P1 clade C (SQTQQEKNEQEL LALDSWKNLWNWFSITNWLWYIK) was derived from the clade C Bw96Bw0502 isolate. P1W is a P1 clade B variant with a W666G mutation and $\mathrm{P} 1-5 \mathrm{~W}$ with all five Ws mutated to $\mathrm{G}$. The scramble peptide sequence comprised the same set of amino acids found in P1 clade B but organized in a random manner (9). Peptides were synthesized with a purity $>95 \%$ by Biopeptide Co., Inc (San Diego, CA) or United BioSystems (VA, USA).

\section{Cells}

Nasal RPMI 2650 cells (isolated from the human nasal septum, squamous cell carcinoma, ATCC) were grown in MEM $\alpha$ (Minimum Essential Medium $\alpha$, Thermo Fisher) supplemented with $10 \%$ fetal calf serum (FCS, Eurobio, Courtaboeuf, France) and $1 \%$ penicillin/streptomycin.

Primary human nasal epithelial cells (HNECs, purchased from PromoCell, Heidelberg, Germany) were isolated from nasal septum or adenoids of healthy donors. Cells from two independent donors were obtained. HNECs were cultured in airway epithelial cell basal medium (PromoCell) and supplemented with airway epithelial cell growth SupplementMix (PromoCell) and only cells from passages 2 to 6 were used.

Monocyte-derived DCs (DCs) were generated from primary human monocytes obtained from PBMCs (purity >98\%) as described $(11,20)$. In brief, human peripheral blood mononuclear cells (PBMCs) were separated from healthy donors blood (EFS, Paris, France), and monocytes were purified from PBMCs by negative selection according to the manufacturer's instructions (StemCell Technologies, France). DCs were obtained by incubating monocytes for 7 days in complete medium containing GM-CSF (100 ng/ml) and IL-4 (10 ng/ml). 
Autologous CD4+ T cells were purified from PMBCs by negative selection according to the manufacturer's instructions (StemCell Technologies, France) (purity >95\%).

\section{Quantitative RT-PCR for TSLP}

The expression of short and long form TSLP was quantified as described $(21,22)$. Briefly, total RNA was extracted using Trizol. Five hundred nanograms of RNA was treated with ezDNase Enzyme (Thermo Fisher) to remove genomic DNA and reverse transcribed into cDNA using the kit SuperScript IV VILO Master Mix according to the manufacturer's instructions (Thermo Fisher). Quantitative PCR was performed using reported primers (21) and the PowerUp SYBR Green Master Mix according to the manufacturer's instructions (Thermo Fisher). Reactions were performed in triplicates, with glyceraldehyde-3-phosphate dehydrogenase (GAPDH) as the internal control. Amplification, data acquisition, and analysis were carried out using the LightCycler 480 Software (Roche, Mannheim, Germany). The levels of TSLP mRNA were normalized to the levels of GAPDH using the $\Delta \mathrm{Ct}$ method (23) and were presented as $2^{-\Delta \mathrm{Ct}}$ values.

\section{MicroRNA Microarray Analysis}

Confluent HNECs in 12 -well plates were stimulated with medium or P1 (clade B, $125 \mu \mathrm{M}$ ) for $6 \mathrm{~h}$ at $37^{\circ} \mathrm{C}$. Total RNA was extracted using Trizol. Before analysis, lfTSLP RNA upregulation was confirmed by qPCR as described above and RNA quality was assessed with Agilent 2100 bioanalyzer according to the manufacturer's instructions (Agilent Technologies). Three untreated and treated paired samples from three independent experiments were analyzed by GeneChip miRNA 4.0 arrays (Affymetrix, Thermo Fisher) containing probes for 2,578 human mature microRNAs and 2,025 premature microRNAs (https://assets.thermofisher.com/TFS-Assets/LSG/brochures/ miRNA_4-0_and_4-1_datasheet.pdf). Potential microRNA targets were analyzed with the Ingenuity Pathway Analysis (IPA) software (Qiagen).

\section{MiR-4485 Quantification and Knockdown}

The quantification and knockdown of microRNA were performed as previously described with some modifications (16). Briefly, total RNA was purified using MinElute PCR Purification Kit (Qiagen), the expression level of miR-4485 was quantified with TaqMan Small RNA Assays (Thermo Fisher). Reactions were performed in triplicates, and U6 was used as endogenous control. In order to knock down miR-4485, 70\% confluent HNEC cells were transfected with anti-miR-4485 inhibitor (67 nM, Qiagen) or mock inhibitor (miSCRIPT inhibitor negative control, $67 \mathrm{nM}$, Qiagen) using Lipofectamine RNAiMAX (Invitrogen) as described by the manufacturer. $36 \mathrm{~h}$ after transfection, miR- 4485 expression, when quantified as described above, was reduced by $50-60 \%$ in anti-miR-4485 transfected cells as compared to anti-miR control ( $\mathrm{n}=3$ independent experiments).

\section{Signaling Inhibitors}

Confluent HNEC cells in 24-well plate were pre-incubated with inhibitors for $1 \mathrm{~h}$ at $37^{\circ} \mathrm{C}$ prior to $\mathrm{P} 1$ treatment. Inhibitors, namely dexamethasone (Dex) a NF- $\kappa \mathrm{B}$ and MAPK inhibitor (used at $100 \mathrm{nM}$ ), and cyclosporin A (CsA) a calcineurin inhibitor (used at $1.5 \mu \mathrm{M}$ ), were from Invivogen and used at the manufacturer's recommended concentrations. ENMD-1068 (PAR-2 antagonist, Enzo Life Science) was used at $50 \mu \mathrm{g} / \mathrm{ml}$ as described $(24,25)$.

\section{Calcium Measurement}

$70-80 \%$ confluent RPMI 2650 or HNEC cells in 24-well plates were loaded with $2 \mathrm{mM}$ Fura-2/AM (Molecular Probes) in basal medium without serum/growth factors for $1 \mathrm{~h}$ at $37^{\circ} \mathrm{C}$. Cells were washed twice with mammalian saline (26), and measurements were performed in complete medium supplied with HEPES $(10 \mathrm{mM})$ and $\mathrm{CaCl}_{2}(2 \mathrm{mM})$ as described (26). Images were acquired with an inverted fluorescence microscope (Observer Z1, Zeiss, Germany) and analyzed with MetaMorph software (27). Calcium was measured every $5 \mathrm{~s}$ by video fluorescence imaging. Results were expressed as $340 \mathrm{~nm}$ to $380 \mathrm{~nm}$ fluorescence ratio and normalized to the baseline, i.e. ratio at time zero was set as 1 .

\section{Cytokines and Chemokine Quantification}

TSLP, IL-25/IL-17E, IL-33, IFN- $\gamma$, IL-10, IL-12/23p40, IL-4, IL-5, IL-6, IL-13, TNF- $\alpha$, MMP-9, IL-8/CXCL8, MIP-3 $\alpha /$ CCL2, MCP-1/CCL20, MDC/CCL22, TARC/CCL17, APRIL, and $\mathrm{BAFF}$ were measured in culture supernatants from the indicated experiments with custom multiplex Luminex assays (Bio-techne) according to the manufacturer's instructions. Additionally, the indicated TSLP was measured in culture supernatants by enzyme-linked immunosorbent assay (ELISA) with a limit of detection of $8 \mathrm{pg} / \mathrm{ml}$ (Thermo Fisher) according to the manufacturer's instructions.

\section{DC-EC Co-Culture and DC Activation}

Three DC culture systems were developed. Monocytes derived DCs $\left(5 \times 10^{5}\right.$ cells) were incubated for $24 \mathrm{~h}$ in medium alone and considered as non-mucosal DCs (DCs) or co-cultured with nasal epithelial cell (RPMI-2650 cell line) monolayer in 24-well plate (DC-EC or eduDC systems for $24 \mathrm{~h}$ at $37^{\circ} \mathrm{C}$. In turn, DCs were either further cultured with ECs during P1 stimulation (DCECs) or separated from EC and transferred into a new plate (eduDCs) for further P1 treatment. Subsequently, P1 (clade B, $125 \mu \mathrm{M}$ ) or medium were added to each of the DCs, DC-ECs or eduDCs cultures for $16 \mathrm{~h}$. DCs were collected for surface staining with allophycocyanin (APC)-conjugated anti-CD86, Rphycoerythrin (PE)-conjugated anti-CD83, APC-conjugated anti-TSLPR, PE-conjugated anti-IL-7R $\alpha$ antibodies (all from Bio-Techne). Specific labeling was quantified by flow cytometry using a Guava EasyCyte flow cytometer and the InCyte software (Merck) described (28). Culture supernatants were collected and frozen at $-80^{\circ} \mathrm{C}$ for subsequent cytokine and chemokine analyses.

\section{DC-T Cell Co-Cultures}

DCs and confluent ECs were co-cultured overnight as described above, and DC-EC or eduDC was further incubated with P1 (clade B, $125 \mu \mathrm{M}$ ) or medium for $24 \mathrm{~h}$. Then, DCs were separated and incubated with autologous CD4+ T cells pre-labeled with 
CFSE (Thermo Fisher) according to the manufacturer's instructions, at a ratio of 1:5 (DC/T). After 5 days of culture, CD4+ $\mathrm{T}$ cell proliferation was analyzed by flow cytometry as described $(29,30)$ using Phytohaemagglutinin (PHA) $(5 \mu \mathrm{g} / \mathrm{ml})$ as positive control.

\section{In Vitro Immunization Assay}

In vitro immunization assay was performed as reported (31) with modifications. Briefly, $1 \times 10^{6}$ CD8-depleted PBMCs (Human CD8 Depletion Cocktail, StemCell Technologies, France) were co-cultured for $24 \mathrm{~h}$ with RPMI 2650 cells $\left(1 \times 10^{5}\right)$ pre-seeded in 48-well plates for $48 \mathrm{~h}$. Then, ovalbumin (OVA, EndoFit Ovalbumin, $10 \mu \mathrm{g} / \mathrm{ml}$, Invivogen) alone, OVA together with P1 $(5 \mu \mathrm{M}, 25 \mu \mathrm{M}, 125 \mu \mathrm{M})$, OVA together with P1 mutant (P1mut, $125 \mu \mathrm{M})$, or medium were added to in RPMI 1640 medium supplemented with Non-Essential Amino Acids (NEAA solution, Thermo Fisher), IL-4 (10 ng/mL), IL-2 (10 UI/mL) and 2-mercaptoethanol $(20 \mu \mathrm{M})$ for 7 days.

For the detection of OVA-specific B cells, at the time points indicated, $\mathrm{PMBCs}$ were surface stained with ovalbumin conjugated to fluorescein (OVA-FITC, $20 \mathrm{ug} / \mathrm{ml}$, Thermo), PEconjugated mouse anti-human CD20 (BD Biosciences, CA, USA), APC-conjugated goat anti-human IgA or donkey antihuman IgG (Jackson ImmunoResearch, PA, USA) as indicated by the manufacturer. Specific labeling was quantified by flow cytometry with a Guava EasyCyte flow cytometer (MerckMillipore), and analyzed with the dedicated InCyte software, using the following strategy: CD20+ B cells were first gated and cells double positive for OVA-FITC+ and APC-conjugated antiIgA or anti-IgG were determined as OVA-IgA or IgG-specific Bcells, respectively.

\section{Statistical Analysis}

Data are presented as mean \pm SEM of at least three independent experiments. Statistical significance was analyzed by the twotailed Student's t-test with the GraphPad Prism software.

\section{RESULTS}

\section{P1 Induces TSLP Secretion in Nasal Epithelial Cells by Interacting With Galactosyl Ceramide}

We first investigate whether P1 induced TSLP secretion in nasal epithelium. Therefore, we cultured human nasal epithelial cells (RPMI 2650) with P1 clade B for $2-24 \mathrm{~h}$ at $37^{\circ} \mathrm{C}$ and analyzed the culture supernatants for TSLP secretion. Compared with the medium and scramble peptides used as negative controls, P1 upregulates TSLP secretion in a dose-dependent manner from 2 to $4 \mathrm{~h}$ (Figure 1A). At $125 \mu \mathrm{M}$, when P1 adopts a trimeric oligomerization state (9), P1 induces a significantly higher secretion of TSLP than in a monomeric state (at $5 \mu \mathrm{M}$ and $25 \mu \mathrm{M})$. TSLP secretion occurs rapidly within hours post stimulation reaching a plateau from 4 to $24 \mathrm{~h}$.

Although the $\mathrm{P} 1$ sequence is relatively conserved, in contrast to highly mutated regions of HIV-1 envelope gp120, the P1 sequence varies between HIV-1 clade A that is common in West Africa, clade B that predominates in Europe and the USA, and clade $\mathrm{C}$ that predominates in Africa and China (Figure 1B). Consequently, we next analyzed whether TSLP secretion was restricted to clade $\mathrm{B}$ derived $\mathrm{P} 1$ or would also be stimulated by $\mathrm{P} 1$ derived from clade $A$ and $C$ viruses (Figure 1B). Secretion of TSLP induced by clade A compared to clade B P1 is reduced by $20 \%(41.8 \pm 2.6 \mathrm{pg} / \mathrm{ml}$ for clade A, $52.3 \pm 3.4 \mathrm{pg} / \mathrm{ml}$ for clade B P1 at $125 \mu \mathrm{M}, p<0.05, \mathrm{n}=5$ ) whereas $\mathrm{P} 1$ clade $\mathrm{C}$ failed to induce TSLP secretion. P1 clade C differs from P1 clades B and A by the ELDKW motif, we have previously shown to be determinant in P1 clade B binding to galactosyl ceramide (GalCer), the epithelial HIV-1 receptor $(9,10)$. We thus hypothesized that P1 clades B and A interaction with GalCer initiated TSLP secretion. Accordingly, P1 clade B mutated in W666G (P1W) that fails to interact with GalCer (9) completely loses the capacity to induce TSLP secretion. Furthermore, when the interaction between P1 and GalCer was blocked by pre-incubation with anti-GalCer antibody, TSLP production is entirely blocked, confirming that TSLP secretion is initiated by P1 interaction with GalCer (Figure 1C). Importantly, P1 stimulation also induces primary human nasal epithelial cells (HNECs) to secrete TSLP in a GalCer-dependent manner (Figure 1D).

\section{Long-Form TSLP Is Up-Regulated After P1 Stimulation}

Two transcript variants of TSLP, namely the short (sfTSLP) and the long (lfTSLP) forms, were recently identified (32). The expression of sfTSLP has been suggested to be constitutive and homeostatic, whereas the lfTSLP leads to proinflammatory responses (32). We thus investigated which form(s) of TSLP was up-regulated by P1 stimulation of nasal epithelial cells. When analyzed at the transcriptional level in nasal RPMI and primary HNEC cells, the expression of sfTSLP and lfTSLP differs by a factor $>10^{2}$. Upon P1 stimulation of both nasal RPMI cells and primary HNECs, the level of the sfTSLP transcript remains unchanged (Figure 1E). In contrast upon P1 stimulation, the level of lfTSLP transcription in both nasal RPMI cells and HNECs increased by $1.9(\mathrm{p}=0.02, \mathrm{n}=5)$ and 5.9 -fold ( $\mathrm{p}=$ $0.004, \mathrm{n}=6$ ), respectively, compared to unstimulated cells. Altogether, these results indicate that P1 up-regulates IfTSLP selectively at a transcription level.

\section{P1-Induced IfTSLP Expression Is Regulated by miR-4485, Calcineurin, and PAR-2}

Next, we investigated the intracellular mechanisms leading to TSLP expression after P1 interaction with GalCer. We concentrated on primary HNECs as its increase in lfTSLP transcription level upon P1 stimulation is higher compared to that in nasal RPMI cells (Figure 1E).

We have previously shown that the non-coding microRNA miR-375 controls TSLP expression in primary human foreskin keratinocytes (16), as it does in human intestinal cell lines (33). When tested in primary HNECs, we found that the TSLP secretion induced by $\mathrm{P} 1$ described above is not accompanied 
A

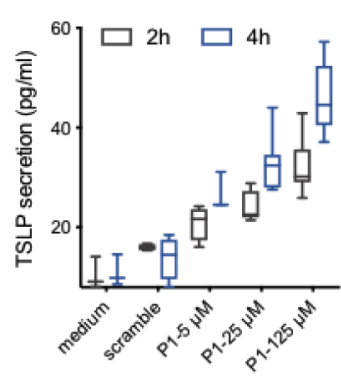

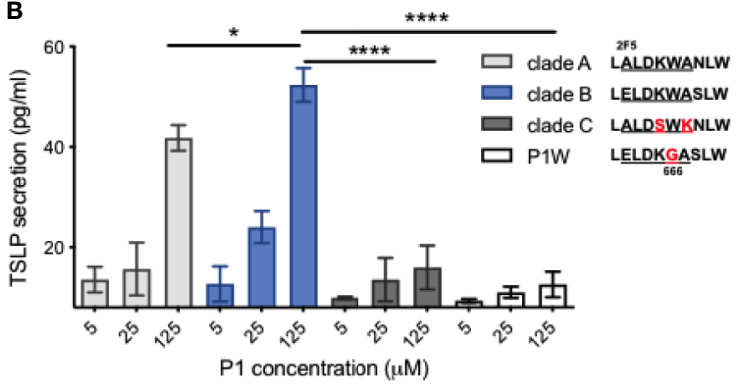

C

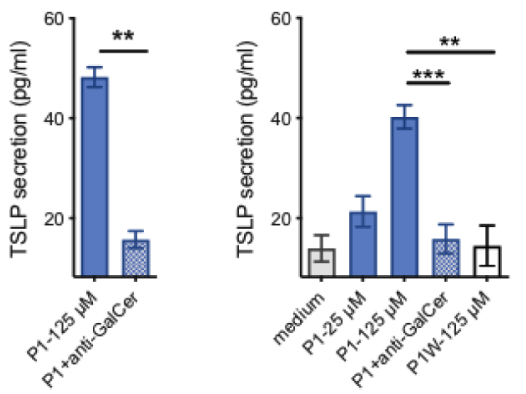

E

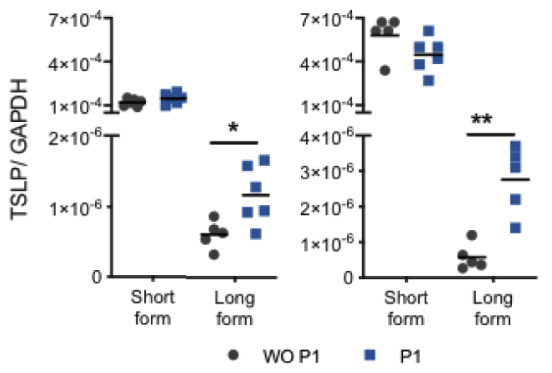

FIGURE 1 | P1 induces TSLP expression in nasal epithelial cells. (A) Confluent RPMI 2650 cells were cultured with P1 peptide (5 $\mu$ M, $25 \mu \mathrm{M}$, $125 \mu$ M) or scramble peptide $(125 \mu \mathrm{M})$, for $2 \mathrm{~h}$ or $4 \mathrm{~h}$. TSLP secretion in culture supernatants was quantified by ELISA. Data are presented as box-and-whisker plots. (B) RPMI nasal cells were cultured with HIV-1 clade (A-C) derived P1 peptides or the mutated P1-5W peptide at increasing concentrations for 4 h. Inset: P1 key amino acids (661-670) corresponding to the broadly neutralizing $2 \mathrm{~F} 5$ and $4 \mathrm{E} 10 \mathrm{lgG}$ epitopes with clade-specific mutations are aligned. (C) RPMI nasal cells were pre-incubated with antigalactosyl ceramide (GalCer) antibody for 30 min at $37^{\circ} \mathrm{C}$ before stimulation with each P1 peptide. (D) Primary HNEC cells were cultured with P1 peptide (5 $\mu \mathrm{M}$, 25 $\mu \mathrm{M}, 125 \mu \mathrm{M})$, with or without anti-GalCer pre-incubation, or P1W peptide (125 $\mu \mathrm{M})$. (E) Relative mRNA expression of the short form (sf) and long form (If) TSLP in RPMI nasal cells (left) and HNEC cells (right). Cells were stimulated with (P1) or without (WO P1) P1 for 4 h, $37^{\circ} \mathrm{C}$. Data in (B-D) are presented as mean \pm SEM ( $n=3-8$ independent experiments. paired student's t-test ${ }^{\star} p<0.05,{ }^{* \star} p<0.01,{ }^{\star \star *} p<0.001,{ }^{* \star * *} p<0.001$ ).

by a change in miR-375 expression. We thus further investigated the microRNA profiles upon nasal epithelial HNEC stimulation by P1 after treatment with or in the absence of P1 for $6 \mathrm{~h}$, comparatively by microRNA array analysis. As a result, 39 microRNAs are differentially expressed with a fold change ranging from 1.3 to 9.15 when $\mathrm{p}<0.05$, including 23 upregulated and 16 down-regulated genes (Figure 2A, Table 1).

Remarkably, in the microRNA array analysis, the highest up-regulated gene upon P1 stimulation is the miR-4485-3p with a $\mathrm{n}>$ nine-fold increase (Table 1). We validated this up-regulation by qPCR resulting in an increase in miR-4485-3p expression by $2.6 \pm$ 0.8 -fold ( $\mathrm{n}=4$ ) upon P1 stimulation (Figure 2B). MiR-4485-3p is a relatively newly described microRNA that is poorly characterized at the experimental level. The only described activity of miR$4485-3 p$ is to regulate mitochondrial functions, suggesting a role in tumor suppression (34). Thus, we first evaluated whether this microRNA controlled TSLP expression. Therefore, primary HNECs were transfected with a specific siRNA to inhibit miR4485-3p expression before P1 stimulation. As a result, knocking down $\mathrm{miR}-4485-3 \mathrm{p}$ by $50-60 \%$ decreases in turn P1-induced TSLP expression by $48 \pm 10 \%(\mathrm{p}<0.01, \mathrm{n}=4)$, compared to cells transfected with a mock inhibitor (Figure 2C).

Bioinformatic analyses were conducted to further elucidate the mechanisms by which P1 modulates all identified
microRNAs and subsequent intracellular signaling pathways. The genes predicted to be targeted by identified microRNAs participate in several signaling pathways, the five principals including G protein-coupled receptor (GPCR)-associated signaling, Nuclear factor of activated T-cells (NFAT) signaling, Rho GDP signaling, Ephrin receptor signaling, and thrombin signaling (Figure 2D).

Corroborating this predictive analysis designating NFAT, GPCR, and thrombin (PAR associated) pathways (35) as the main ones induced by $\mathrm{P} 1$ stimulation, it has been described that in keratinocytes, TSLP production is regulated by $\mathrm{Ca}^{2+}$-dependent NFAT signaling itself triggered by the activation of GPCR protease-activated receptor 2 (PAR-2) (36). Thus, we next evaluated experimentally whether inhibitors specific to these pathways also reduced P1-induced TSLP expression in primary HNECs. Therefore, HNECs were pre-treated with the calcineurin inhibitor Cyclosporine A (CsA) or with the PAR-2 antagonist ENMD-1068 prior to P1 stimulation. Accordingly, TSLP expression was reduced by $67 \pm 4 \%(p<0.001, \mathrm{n}=3)$ upon CsA pre-treatment and by $46 \pm 14 \%(p<0.05, \mathrm{n}=3)$ following ENMD-1068 pre-treatment (Figure 2E). Furthermore, CsA and ENMD-1068 inhibitors also blocked the up-regulation of miR4485-3p (Figure 2B). In contrast, blocking NF- $\kappa \mathrm{B}$ and MAPK with Dexamethasone (Dex) had no effect on TSLP expression 
A

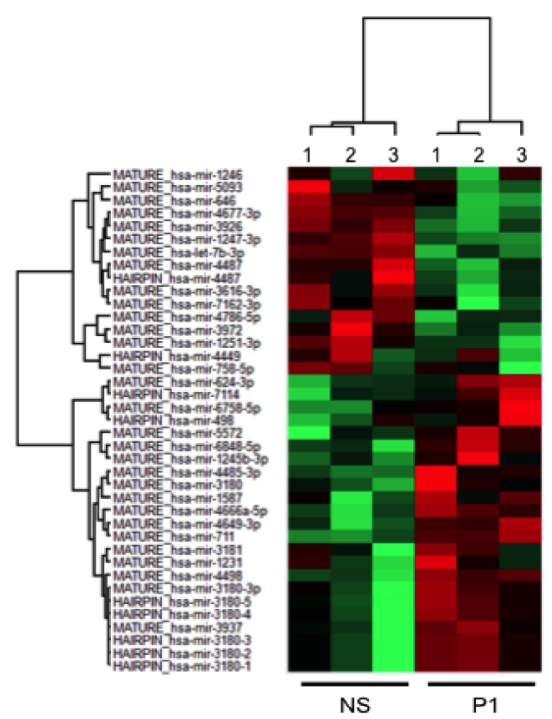

D

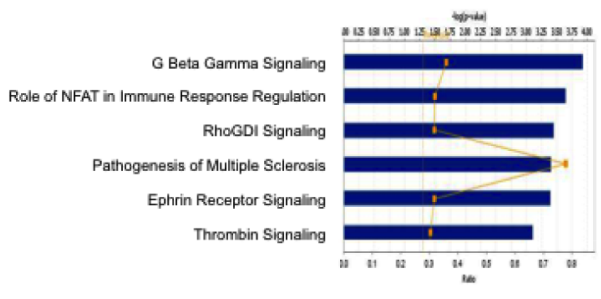

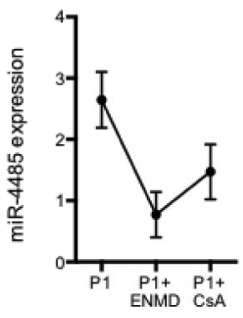

C

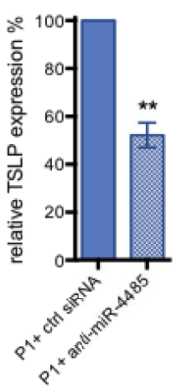

E

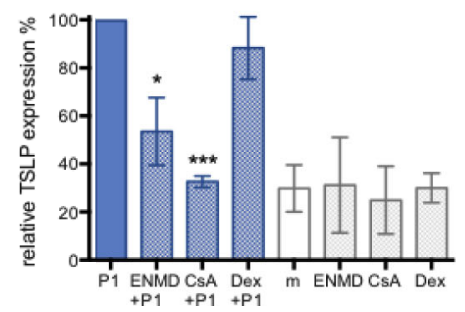

FIGURE 2 | MicroRNA-regulated P1-induced TSLP expression and corresponding signaling pathways in nasal epithelial cells. (A) Heatmap showing 39 differentially expressed miRNAs in P1 stimulated (P1) HNEC cells compared with control unstimulated cells (NS). Only differentially expressed microRNAs with $p$ value $<0.05$ from paired student's t-test, and a cut-off of fold change $>1.3$ are included. $n=3$ independent experiments with HNECs from two different donors. (B) PAR-2 antagonist ENMD-1068 (ENMD) and calcineurin inhibitor cyclosporine A (CsA) decrease the expression of P1-induced miR-4485 as measured by qPCR. (C) miR-4485 knockdown in HNECs by siRNA transfection inhibited P1-induced TSLP expression, measured by qPCR, by about 50\%. (D) Top six target pathways predicted by Ingenuity Pathway Analysis sorted by their $p$ values. The ratio presented is defined as the number of the differentially miRNAs found in the P1-treated cells over the total number of miRNA involved in each of the pathway. (E) P1-induced TSLP expression, measured by qPCR, is significantly reduced in the presence of CsA, an inhibitor of NFAT and ENMD, an antagonist of PAR-2, but not by Dexamethasone (Dex), an inhibitor of NF- $\mathrm{KB}$ and MAPK. m: medium. Data are presented as mean \pm SEM $n \geq 3$ independent experiments paired student's t-test ${ }^{\star} p<0.05,{ }^{\star \star} p<0.01,{ }^{* \star *} p<0.001$ ).

(Figure 2E). These results provide direct and indirect evidence that miR-4485-3p, calcineurin, and PAR-2-mediated signaling tightly correlate with P1-induced TSLP expression.

To further confirm that P1 activates calcineurin, we investigated whether, in nasal epithelial cells, P1 induces calcium fluxes that generally cause calcineurin activation (37). Accordingly, using fluorescent dye Fura-2/AM imaging technology, we observed in both nasal RPMI cells and primary HNEC cells that P1 treatment induces an immediate extracellular calcium influx in a concentration-dependent manner $(125 \mu \mathrm{M}$ vs $25 \mu \mathrm{M}$ of $\mathrm{P} 1, \mathrm{n}=3)$ (Figures $3 \mathrm{~A}, \mathbf{B}$ ). In contrast, treatment with control peptides (P1-5W mutant and $\mathrm{P} 1$ clade $\mathrm{C}$, both at $125 \mu \mathrm{M}$ ) fails to raise the calcium level significantly.
Together, these data indicated that in nasal epithelial cells, P1stimulated TSLP expression is regulated by miR-4485 via a Ca ${ }^{2+}$. dependent NFAT signaling pathway through the interaction with PAR-2 receptor.

\section{P1 Further Stimulates Epithelial Secretion of MMP-9, CCL20, CCL2, and IL-10}

We next investigated whether, in addition to TSLP, P1 could stimulate epithelial secretion of additional immune factors prone to attract antigen presenting cells (APCs). Therefore, nasal RPMI cells where incubated with P1 $(125 \mu \mathrm{M})$ and after $24 \mathrm{~h}$, the cell culture medium was analyzed for interleukin (IL)-25/IL-17E, IL-33, IFN- $\gamma$, IL-10, IL-12/23p40, IL-4, IL-5, IL-6, IL-13, TNF- $\alpha$, Matrix metalloproteinase 9 ((MMP-9), IL-8/CXCL8, MIP-3 $\alpha$ / 
TABLE 1 | MicroRNAs induced (left) or repressed (right) in HNECs treated with P1.

\begin{tabular}{|c|c|c|c|c|c|}
\hline gene ID & fold (P1/n.s.)** & p value $* * *$ & gene ID & fold (P1/n.s.) & $p$ value \\
\hline hsa-mir-4485-3p & 9.15 & 0.049 & hsa-mir-3972 & -1.95 & 0.020 \\
\hline hsa-mir-5572 & 1.95 & 0.030 & hsa-mir-4487 & -1.77 & 0.013 \\
\hline hsa-mir-3180 & 1.67 & 0.030 & hsa-mir-4786-5p & -1.62 & 0.024 \\
\hline hsa-mir-3937 & 1.65 & 0.035 & hsa-mir-1246 & -1.56 & 0.012 \\
\hline hsa-mir-1587 & 1.56 & 0.002 & hsa-mir-5093 & -1.53 & 0.026 \\
\hline hsa-mir-6758-5p & 1.56 & 0.012 & hsa-mir-7162-3p & -1.52 & 0.018 \\
\hline hsa-mir-711 & 1.54 & 0.001 & hsa-let-7b-3p & -1.48 & 0.030 \\
\hline hsa-mir-3180-3p & 1.54 & 0.023 & hsa-mir-3926 & -1.47 & 0.003 \\
\hline hsa-mir-3180-5* & 1.47 & 0.011 & hsa-mir-758-5p & -1.40 & 0.011 \\
\hline hsa-mir-3180-4* & 1.46 & 0.021 & hsa-mir-4677-3p & -1.40 & 0.004 \\
\hline hsa-mir-3180-1* & 1.44 & 0.039 & hsa-mir-3616-3p & -1.40 & 0.003 \\
\hline hsa-mir-3180-2* & 1.44 & 0.039 & hsa-mir-1247-3p & -1.36 & 0.037 \\
\hline hsa-mir-3180-3* & 1.44 & 0.039 & hsa-mir-646 & -1.35 & 0.016 \\
\hline hsa-mir-4666a-5p & 1.41 & 0.040 & hsa-mir-4449* & -1.33 & 0.023 \\
\hline hsa-mir-7114* & 1.38 & 0.041 & hsa-mir-4487* & -1.32 & 0.046 \\
\hline hsa-mir-3181 & 1.37 & 0.042 & hsa-mir-1251-3p & -1.31 & 0.010 \\
\hline hsa-mir-6848-5p & 1.35 & 0.026 & & & \\
\hline hsa-mir-1231 & 1.34 & 0.013 & & & \\
\hline hsa-mir-4649-3p & 1.33 & 0.035 & & & \\
\hline hsa-mir-4498 & 1.33 & 0.036 & & & \\
\hline hsa-mir-624-3p & 1.31 & 0.026 & & & \\
\hline hsa-mir-1245b-3p & 1.31 & 0.045 & & & \\
\hline hsa-mir-498* & 1.31 & 0.007 & & & \\
\hline
\end{tabular}

Hairpin format pre-mature microRNA; ${ }^{* *}$ n.s., non stimulated; ${ }^{* * *} p$ values were calculated from paired t-test.

CCL2, MCP-1/CCL20, MDC/CCL22, TARC/CCL17, APRIL, and BAFF by Luminex technology. As a result, P1 selectively induced the secretion of MMP-9, CCL20, CCL2, and IL-10 (Figure 3C). Furthermore, as observed for P1-induced TSLP secretion, $\mathrm{P} 1 \mathrm{~W}$ and $\mathrm{P} 1$ clade $\mathrm{C}$ were unable to stimulate significant MMP-9, CCL-20 CCL2, or IL-10 production. Together with TSLP (16), this set of immune factors could facilitate recruitment of APCs to the mucosal surface for initiation of an immune response, since CCL20 and CCL2 chemo-attract macrophages and immature DCs and MMP-9 degrade the extracellular matrix and facilitate the migration of immune cells in or out the epithelium. Treg cells have IgAinducing functions and require RA, TGF-b1, IL-10, and TSLP from the intestinal epithelial cells and DCs. So, we assumed IL-10 released from either EC or DC may contribute to IgA class switching (Gutzeit, Magri, et al., 2014).

\section{P1 Activates Human Dendritic Cells in a Nasal Mucosal Model}

APCs link the innate and adaptive immune systems and determine the polarization of the immune responses. APCs are thus a key target in vaccine and adjuvant development (38). DCs being the most abundant APCs in airway mucosa (39), we further investigated mucosal DC responses to P1.

It has been suggested that mucosal DCs display unique functions due to the local microenvironment, especially at mucosal level (40). In particular, mucosal DCs modulate their functions by interacting with epithelial cells (ECs) including via epithelial secretion of $\operatorname{TSLP}(33,41)$. Thus, we established a simplified mucosal DC model, by co-culturing DCs and nasal ECs (RPMI-2650 cell line), thereby mimicking the nasal mucosal environment as depicted in Figure 4A. DCs were first 'educated' by a $24 \mathrm{~h}$ co-culture with ECs. Subsequently, these 'educated' DCs were either maintained in culture with ECs and referred to as DC-EC, or separated from the epithelium and referred to as eduDC. Alternatively, DCs only cultured with medium represented 'non-mucosal' DCs.

Each type of DCs was stimulated with P1 overnight, and the expression of maturation markers was assessed by flow cytometry. Compared to untreated cells, P1-treated mucosal DCs, either DC-EC or eduDCs, show a significant upregulation of co-stimulatory molecules CD83 (Figure 4B) and CD86 (Figure 4C). In contrast, P1 has no effect on 'non-mucosal' DCs. Surprisingly, P1 also significantly enhanced the expression of TSLP receptor, with both chain TSLP-R (Figure 4D) and IL-7R $\alpha$ (Figure $4 \mathbf{E}$ ) being up-regulated on the DCs in all three models.

The cytokine and chemokine secretion profiles were also studied in these models, comparatively. Compared with non-mucosal DCs, P1 induces a significant increase in IL-6, IL-8, IL-10, CCL20, CCL22, and MMP-9 secretion in eduDC and DC-EC models as well as that of TSLP secretion, although more modest. In contrast, IFN- $\gamma$ secretion remains unchanged upon P1-stimulation or slightly decreases in DC-EC (Figure 5A). In addition, several cytokines, such as IL-25, IL-33, IL-4, IL-5, remain undetectable whatever the model, whereas others, such as IL-12, IL-13, CCL2, CCL17, TNF- $\alpha$, APRIL, and $\mathrm{BAFF}$, are secreted equally in all three models.

Activated DCs are known to stimulate T-cell proliferation to initiate an adaptive immune response, both in vivo and in vitro $(15,29,30)$. Therefore, we further assessed if P1 activated mucosal DCs could promote $\mathrm{T}$ cell proliferation. As a result, P1 primed eduDCs induced the proliferation of autologous CD4+ T cells, whereas treatment with control peptides (P1W mutant and P1 clade $\mathrm{C}$ ) or P1 stimulation on CD4+ T cells alone has no effect 
A

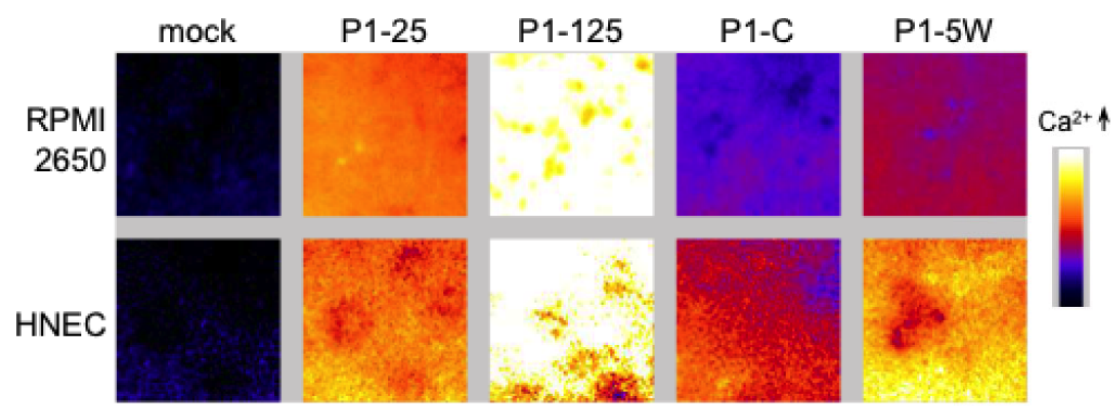

B
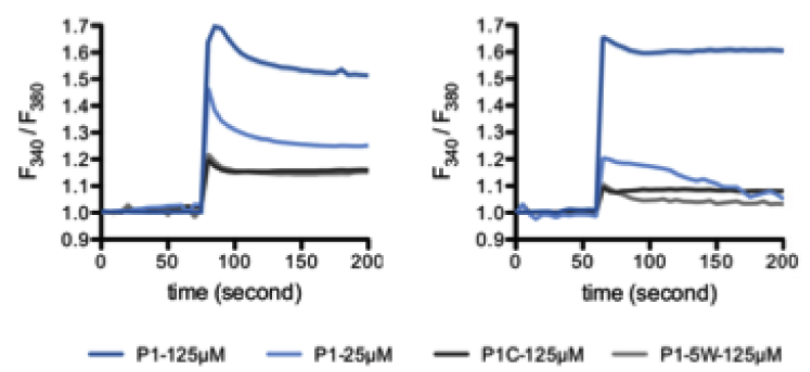

C

MMP-9

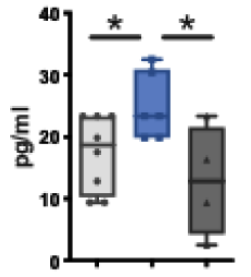

CCL20

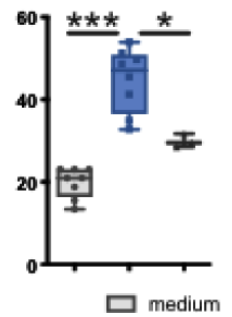

CCL2

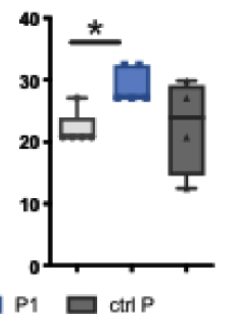

IL-10

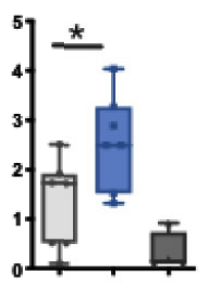

FIGURE 3 | P1 induces calcium flux and cytokine/chemokine secretion in nasal epithelial cells. (A, B) Calcium fluxes in response to P1 stimulation. (A) Representative images of Fura-2/AM loaded epithelial cells treated with medium (mock), P1 (5 $\mu \mathrm{M}, 25 \mu \mathrm{M}, 125 \mu \mathrm{M}), \mathrm{P} 1$ of clade C (P1-C, $125 \mu \mathrm{M})$, and mutated $\mathrm{P} 1$ (P1-5W, $125 \mu \mathrm{M})$. (B) Calcium was measured every $5 \mathrm{~s}$ by video fluorescence imaging and displayed as the ratio of 340 and $380 \mathrm{~nm}$ excitation signals. Representative trace out of $n=3$ independent experiments of calcium fluxes in Fura-2/AM loaded epithelial cells (left RPMI, right HNEC cells) treated with P1 and other indicated peptides. (C) Cytokine and chemokine release from nasal epithelial cells upon P1 stimulation. P1 elicits MMP-9, CCL20, CCL2, IL-10 secretion in nasal epithelial cells. Cells were incubated with medium, P1 (clade B, $125 \mu \mathrm{M}$ ) or control peptide: scramble P1, P1-5W or P1 clade C, $125 \mu \mathrm{M}$ (ctrl P) for 24 h. MMP-9, CCL20, CCL2, IL-10 secretions were measured by multiplex Luminex assay. Data are presented as mean \pm SEM (A, B) Data are presented as box-and-whisker plots from $n=5$ independent experiments; paired student's t-test ${ }^{\star} p<0.05,{ }^{\star \star *} p<0.001$.

(Figure 5B). Similar results were observed with DC-ECs, in agreement with the similar cytokine profiles between DC-EC and eduDC as described above.

Altogether, these results show that P1 activates mucosal DCs specifically, resulting in Th2 cytokine and chemokine secretion, and in $\mathrm{CD} 4+\mathrm{T}$ cell proliferation.

\section{P1 Acts as an Adjuvant to Stimulate Antigen-Specific Humoral Responses In Vitro}

Finally, given that $\mathrm{P} 1$ induces various immunomodulatory effects in mucosal cells involved in vaccination at the nasal site, as described above, we investigated whether P1 was able to act as an adjuvant. Using an in vitro immunization model with human PBMCs, the capacity of P1 to trigger a humoral immune response against a well-characterized antigen, namely ovalbumin (OVA), was evaluated.

In vitro immunization assays have been used to produce specific monoclonal antibodies using a defined antigen complemented with an adjuvant (42). Here, we establish a mucosal immunization model adapted from $(29,31,43)$ and using mucosal DCs based on our results presented above. OVA were selected as the model antigen. Therefore, human CD8depleted PBMCs ( $\mathrm{n}=5$ independent donors) were co-cultured with RPMI 2650 cells for one day to educate DCs, and prior to addition of either medium, OVA, OVA plus P1 mutant (P1mut, 
A

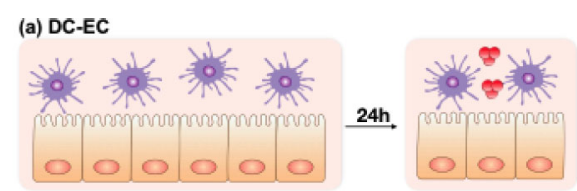

(b) eduDC

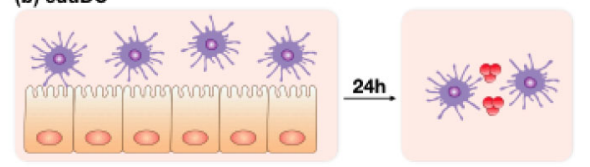

(c) DC

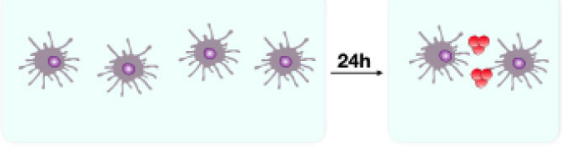

B

C

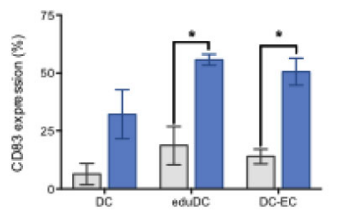

D
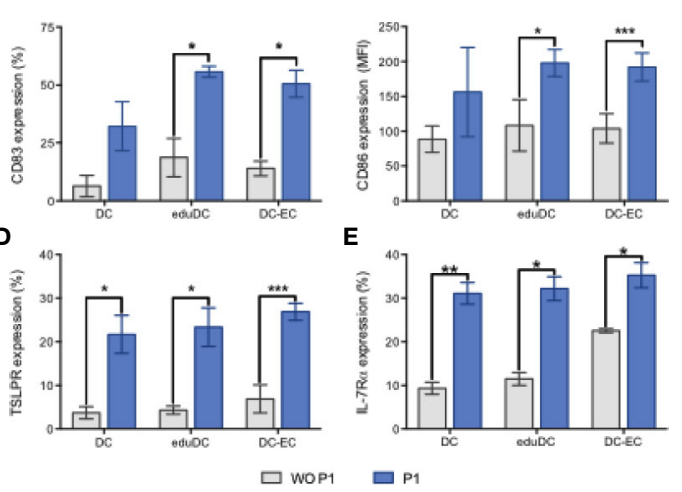

E

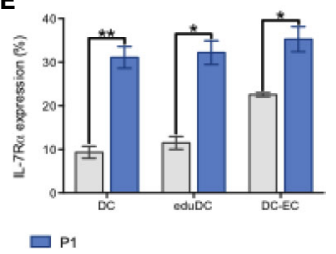

FIGURE 4 | P1 induces mucosal dendritic cell (DC) maturation. (A) Experimental models of mucosal DC activation by P1. Experimental model evaluating (a) mucosal DC generated by continuous contact between epithelial (EC) and DC (DC-EC): DCs, co-cultured with EC monolayer (RPMI2650 cell line) for $24 \mathrm{~h}$, were treated with $\mathrm{P} 1$ or medium for an additional 16 h; (b) mucosal DC generated by an initial education by EC following a time-limited contact between EC and DC (eduDC): after co-cultured with ECs for $24 \mathrm{~h}$ as above, DCs were separated from EC and subsequently treated with P1 or medium for an additional $16 \mathrm{~h}$. (c) non-mucosal DC (DC): DCs were cultured alone with medium for $24 \mathrm{~h}$ prior to stimulation by $\mathrm{P} 1$ or medium for 16 h. (B-E) P1 activates DCs by up-regulating the expression of surface markers. Expression of CD83 (B), CD86 (C), TSLPR (D), IL-7Ra (E) on DCs obtained after culture within the three models described in (A) and quantified by flow cytometry with (blue bars) or without (WO) (gray bars) P1 stimulation. CD86 expression is shown as mean fluorescence intensity (MFI). Data are presented as mean \pm SEM $(n>3$ independent experiments; paired student's t-test ${ }^{*} p<0.05$, ${ }^{\star \star} p<0.01,{ }^{\star \star *} p<0.001$ ).

$125 \mu \mathrm{M})$ or OVA plus P1 $(5 \mu \mathrm{M}, 25 \mu \mathrm{M}, 125 \mu \mathrm{M})$ for seven more days. OVA-specific B cells were quantified by flow cytometry using FITC-conjugated OVA and anti-CD20-PE to gate on OVA-specific B cells. The Ig isotype of surface B cell receptor (BCR) was next characterized by APC-conjugated anti-human-IgA or anti-human-IgG. As shown in Figure 6, OVA alone, similarly to medium, failed to induce OVA-specific specific B cells, whereas in the presence of P1, OVA-specific B cells were detected. At $5 \mu \mathrm{M}$

and $25 \mu \mathrm{M}$, the concentration at which $\mathrm{P} 1$ remains in the monomeric state, induction of OVA-specific B cells is very limited, whereas, at $125 \mu \mathrm{M}, \mathrm{P} 1$ significantly enhances OVA recognition by $\mathrm{B}$ cells due to surface expression of OVA-specific IgA and IgG isotypes. Similar results were obtained when B cells were stained with anti-CD19-PE. Importantly, in the absence of nasal epithelial cells during the in vitro immunization, $\mathrm{P} 1$ is not able to induce OVA-specific B cells. Within the culture supernatants of day 7, OVA-specific antibody secretion could not be detected by ELISA, most likely because blasts were not formed at this early time point of the immunization and in agreement with the detection of OVA-specific IgA and IgG at the B cell surface, prior to blast differentiation. Hence, P1 appears to act as an adjuvant by promoting the expression of antigen-specific BCR on B cells, which may need additional signals to develop into plasma cells.

\section{DISCUSSION}

In contrast to intensively used bacterial adjuvants, the beneficial immune properties of viral components have been mostly overlooked. Although viruses are commonly considered to trigger PRRs (Pattern recognition receptors) by their genetic materials, viral envelope proteins have also been reported to initiate local inflammation via the interaction with TLR-2 and TLR-4 (44-47). These motifs are critical for virus transmission but might also be used to improve vaccine uptake and efficacy.

Here, we reported for the first time about the adjuvant activity of a viral membrane protein and explored the corresponding immunological mechanisms. Therefore, we have characterized the immuno-modulatory properties of $\mathrm{P} 1$, a conserved peptide from the HIV-1 glycoprotein gp41 we have previously shown to be a powerful vaccine antigen providing full protection against mucosal HIV infection following intra-nasal immunization (8) and determined its stimulatory activities in human nasal epithelial cells and dendritic cells, the two major targets of nasal vaccination.

Nasal epithelial cells are the first cells encountered by the vaccine applied nasally and are recognized to influence the initiation, regulation, and maintenance of mucosal innate and adaptive immune responses via epithelial-derived factors, such as TSLP $(33,41)$. The P1-induced TSLP secretion by nasal epithelial cell shown here could thus constitute the initial adjuvant activity achieved by $\mathrm{P} 1$ used in a nasal vaccine formulation. TSLP offers several advantages for stimulation of the local immunity and induction of specific antibody production, two main goals of mucosal vaccination (18). In particular, TSLP affects polarization towards Th2 immune responses, promotion of IgA class switching, and mediates the generation and maintenance of memory $\mathrm{T}$ cells (48). Hence, the introduction of TSLP in a vaccine formulation applied mucosally strongly stimulates the IgA response (19), and TSLP has demonstrated adjuvant activity in a recent HIV-1 vaccine study with intra-nasal administration in the mouse model (17). Recent studies by us and others demonstrated that TSLP is secreted during the initial steps of HIV-1 entry in the genital mucosa 
A
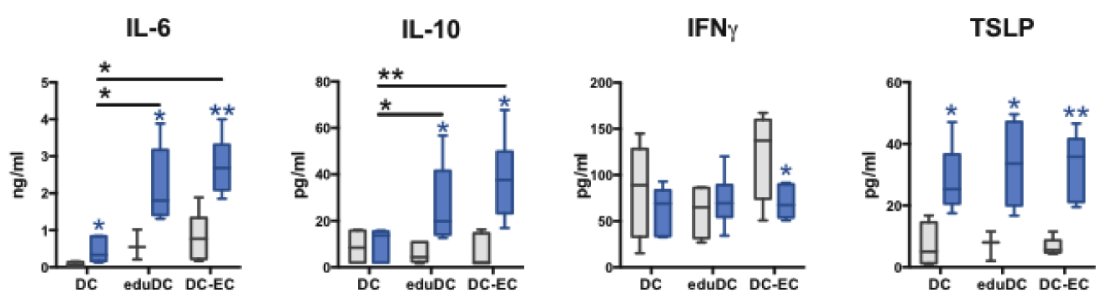

IL-8

CCL-20

CCL-22

MMP-9
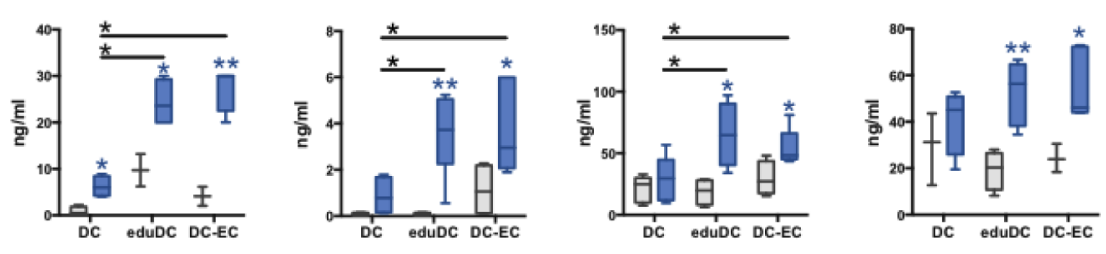

口 WoP1 $\square$ P1

\section{B}
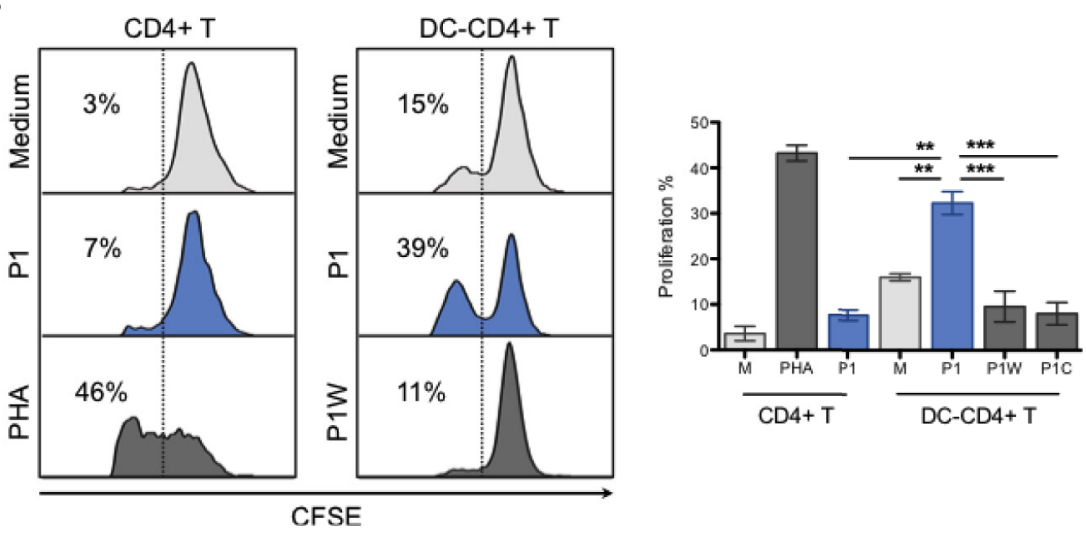

FIGURE 5 | P1 stimulation of mucosal DCs results in CD4+T cell proliferation. (A) Cytokine and chemokine production of P1-treated DC models. IL-6, IL-10, IFNg, TSLP, IL-8, CCL-20, CCL-22, and MMP9 were quantified by a multiplex Luminex assay. The blue star $\left({ }^{*}\right)$ indicates significant differences $(p$ values $<0.05$, student's t-test) in each model between P1 (blue bars) and mock (gray bars) treatments, whereas the black star ( $\left.{ }^{*}\right)$ compares P1-treated cells in different models. Data are presented as box-and-whisker plots from $n>3$ independent experiments, $p$ values $<0.05$, paired student's t-test). (B) P1-treated mucosal DCs stimulate the proliferation of CD4+ T cells. CD4+ T cell proliferation was determined by flow cytometry according to CFSE intracellular concentration. Data are represented as proliferating CD4+ T cells as \% of total CD4+ T cells. Phytohaemagglutinin (PHA) was used as positive control. Data are presented as mean \pm SEM $(n>3$ independent experiments; paired student's t-test ${ }^{*} p<0.05,{ }^{* \star} p<0.01,{ }^{\star \star *} p<0.001$ ).

following gp120-dependent activation of the TLR-4 pathway through NF- $\kappa$ B signaling $(15,16)$. Based on the considerable efficacy of our gp41-subunit vaccine comprising virosome, an adjuvant-free vaccine carrier, coupled to gp41 subunits used as antigen, and administered nasally, we decided to investigate whether our vaccine antigen, the gp41-subunit P1, could also stimulate TSLP and thus acts as a mucosal adjuvant.

However, nasal epithelial cells differ from genital ones and thus respond differently to pathogens they are associated with. Hence, nasal epithelial cells cannot be activated by LPS via TLR-4 (49). Accordingly, our study revealed that P1 stimulates TSLP production in nasal epithelial cells by interacting rather with GalCer, the HIV mucosal receptor expressed on epithelial cells and dendritic cells (9-11).
We further studied the in-depth mechanism stimulated by P1 interaction with GalCer to identify the cellular regulator of TSLP expression in nasal epithelium. As TSLP secretion by various stimuli is regulated by miRNA $(16,33)$, we first studied the microRNA transcriptome selectively induced in nasal epithelial cells upon P1 stimulation. We identified miR-4485 as the main miRNA that is differentially increased in nasal cells upon P1 stimulation. Accordingly, blocking miR-4485 abolished P1induced TSLP secretion. Further prediction-based systematic analysis based on targets of identified miRNAs computed that the PAR-2 associated NFAT pathway was modulated. PAR-2 plays a key role in TSLP release, particularly the PAR-2/ORAI1/ NFAT calcium pathway that has been reported to regulate TSLP production in keratinocytes (36). Here, we experimentally 


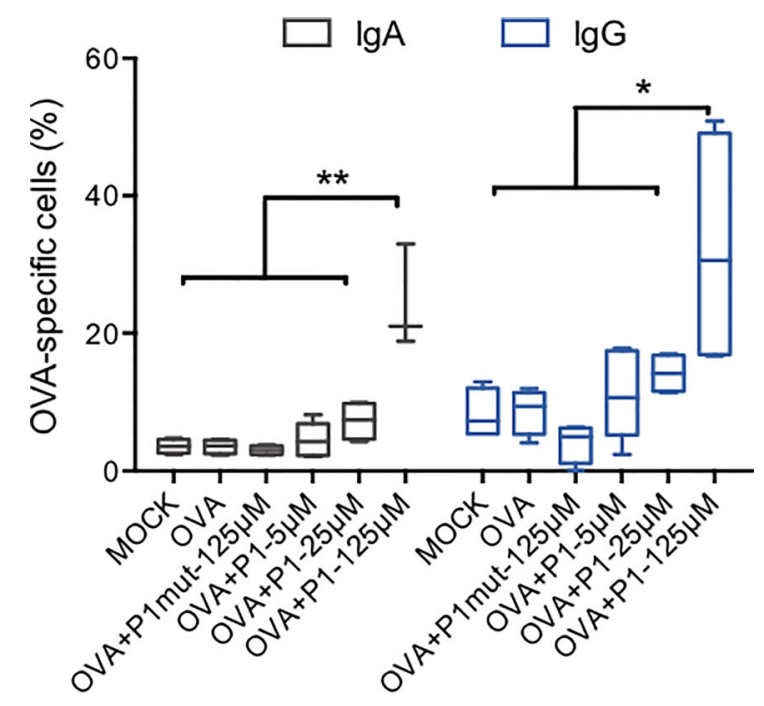

FIGURE 6 | In vitro immunization with ovalbumin adjuvanted by P1 peptide. CD8-depleted PBMCs were co-cultured with RPMI nasal cell monolayer for $24 \mathrm{~h}$ prior to addition of OVA as an antigen, adjuvanted by $\mathrm{P} 1$ at three concentrations or in the presence of mutated P1 (P1mut), in the absence of antigen or adjuvant as negative controls. CD20+ B cells expressing OVAspecific IgA or lgG were quantified by flow cytometry using anti-CD20-PE, FITC-conjugated OVA, and APC-conjugated anti-human-lgA or anti-human-lgG. Data are presented as box-and-whisker plots from $n=5$ independent donors; paired student's t-test ${ }^{\star} p<0.05,{ }^{\star \star} p<0.01$.

established the capacity of P1 to stimulate the PAR-2/ORAI1/ NFAT calcium pathway in nasal epithelial cells. Hence, P1induced TSLP secretion is blocked by PAR-2 and calcineurin specific inhibitors, and $\mathrm{P} 1$ induces an intracellular calcium influx. Furthermore, in addition to TSLP, P1 stimulates the specific secretion of CCL20, CCL2, IL-10, and MMP-9 in nasal epithelial cells. In line with these results, MMP-9 secretion has been shown to be triggered by PAR-2 activation (50).

Our results indicate that $\mathrm{P} 1$ can induce TSLP production when interacting with the nasal epithelium thus probably also during nasal vaccination. However, the pathway induced by the gp41-subunit P1 differs completely from that elicited by gp120 within the HIV-1-envelope spike during HIV-1 sexual transmission at genital sites $(15,16)$. Regarding its role in the nasal vaccination process, TSLP produced at the inductive site may imprint mucosal immune cells, such as DCs, so that these cells acquire an innate memory (51), prior to circulating towards the effector site at the genital mucosa, as proposed (3). Consequently, during the very first contact with HIV-1 at the genital mucosa-also the vaccine effector site-these imprinted immune cells could receive from HIV-1 envelope a signal to induce TSLP, and in turn rapidly mobilize vaccine induced memory cells to prevent infection.

On the non-lymphoid area of the mucosal surface, submucosal DCs act as sentinels for monitoring 'danger signals' such as cytokines and chemokines induced by antigen stimulated epithelial cells. Upon activation, these DCs may penetrate epithelial tight junctions via trans-epithelial dendrites and uptake antigens, as characterized in the nasal epithelium (30, 52). DCs are generally considered as the major target of TSLP (53). In a previous study using TSLP as an adjuvant, nasal application at the nasal epithelial surface was sufficient to activate sub-mucosal DCs, suggesting that TSLP-induced signal is able to cross the epithelial barrier (17). Therefore, we further investigated the modulatory effect of P1 on mucosal DCs, by using a nasal ECs and DCs co-culture model to mimic the nasal mucosal environment and cell-cell contacts.

Our data reveal that P1 stimulates the expression of the costimulatory factors CD83 and CD86 on mucosal DCs, as well as the TSLP receptor. Considering that TSLP regulates Th2 polarization, we investigated the cytokine and chemokine profile stimulated by $\mathrm{P} 1$ in mucosal DCs. Our data showed that in mucosal DCs, P1 induced IL-6, IL-10, and reduced IFN- $\gamma$, corresponding to an anti-inflammatory Th2 response and enhancing IgA class switching. IL-8, CCL20, and CCL22 are also produced and, in turn, could induce the recruitment of immune cells for the initiation of the adaptive immune response (macrophages, lymphocytes, monocytes) to the mucosal stroma. The last factor secreted upon P1 stimulation is MMP-9 that facilitates the migration of immune cells by degrading the extracellular matrix. In addition, P1-modulated mucosal DCs lead to autologous CD4+ $\mathrm{T}$ cell proliferation. Thus, our results indicate that $\mathrm{P} 1$ activates mucosal DCs to release robust Th2 cytokines and chemokines and therefore might promote the mucosal humoral response.

TSLP produced by ECs is expected to mediate crosstalk with DCs leading to Th2 polarization of the immune response. However, we surprisingly found that ECs are not the only source of TSLP in our mucosal models. Mucosal DCs obtained in our two models, namely DC-EC and eduDC, react similarly to P1 stimulation. In particular, P1 induces a similar level of TSLP secretion and increased the expression of TSLP receptor, two elements that might form together a TSLP autocrine loop, in turn, amplifying the TSLP-induced signal. This autocrine loop is actually consistent with the clinical studies reporting that TSLP secretion and TSLP receptor expression are augmented simultaneously upon EC activation $(54,55)$. Such autocrine TSLP loop in DCs would sustain the source of TSLP and help to maintain the DC-epithelial crosstalk. Accordingly, we found that the 'education' induced by the interaction with ECs and its specific microenvironment provide unique functional features to mucosal DCs upon P1 stimulation characterized by a different immune profile compared to blood DCs. This is consistent with previous reports indicating that the immunological consequences subsequent to an adjuvant stimulation are primarily dependent on the environment in which the adjuvant is applied $(41,56)$. Further studies will be necessary to better understand the mechanisms involved in the epithelial 'education' and on P1-induced DC activation pathways.

As a final step in the characterization of $\mathrm{P} 1$ adjuvant function, we showed that P1 induces antigen-specific IgG and IgA at the surface of $\mathrm{B}$ cell in an in vitro immunization assay. Previous studies reported that using PBMCs depleted in immunosuppressive cells, an antigen-specific immune response can be elicited by antigen sensitization in the presence of cytokines 


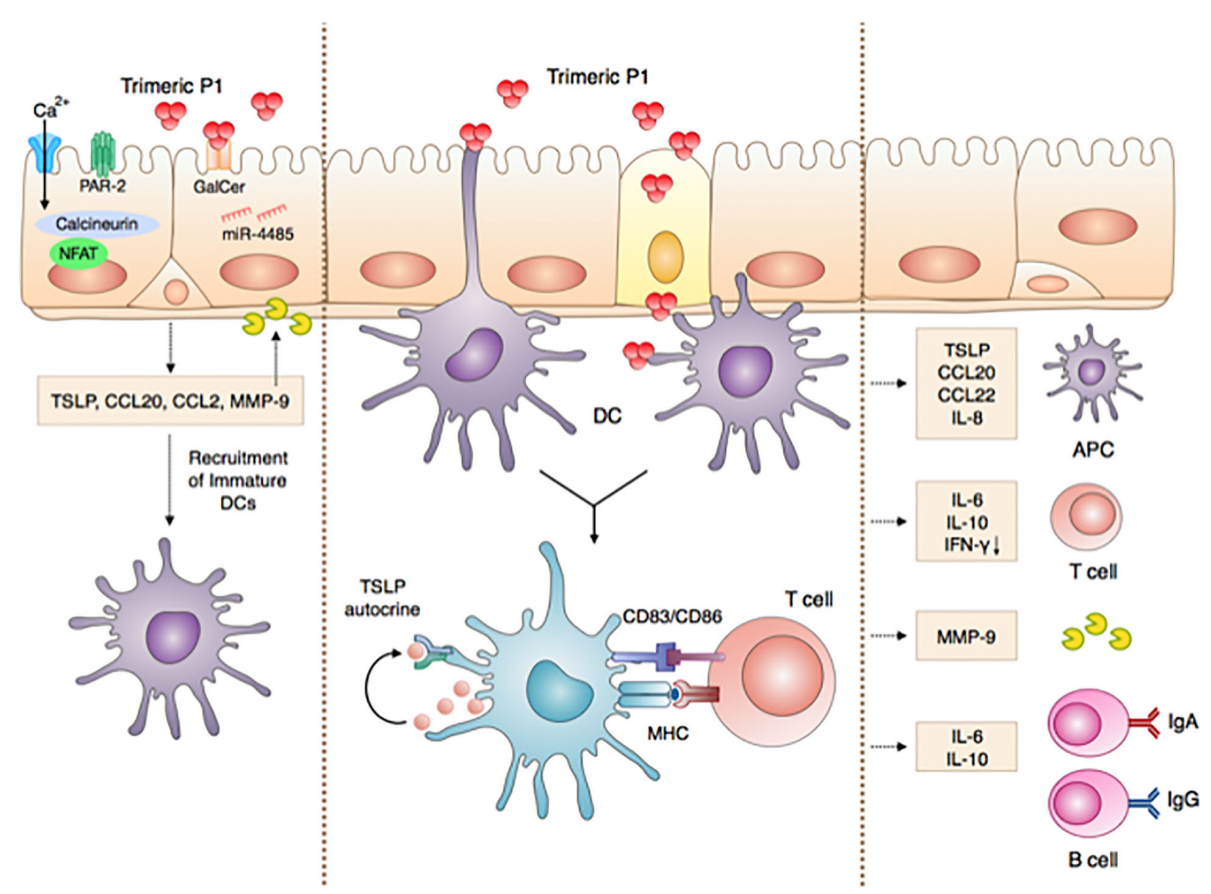

FIGURE 7 | 'Chain of events' summarizing the P1 immunomodulatory adjuvant activity in initiating innate and adaptive immune responses at the nasal mucosa. Step 1: P1 activates nasal epithelia cells to produce TSLP by interacting with GalCer, activating, in turn, miR-4485, calcium influx, PAR-2 and calcineurin, as well as the secretion of CCL20, CCL2, MMP-9, and IL-10. These cytokines and chemokines initiate the very first steps of the immune response and mediate the crosstalk between ECs and immune cells such as DCs. Step 2: P1 interacts with mucosal DCs, thereby enhancing the expression of co-stimulatory markers and TSLP receptor in an autocrine loop and favoring DC maturation. As a result, P1-activated mucosal DCs initiate an adaptive immune response, by eliciting chemokine release that in turn can recruit adaptive immune cells and cytokine secretions. Consequently, adaptive immunity is polarized into a Th2 and IgA preferential response. Step 3: Finally, P1-stimulated DCs promote CD4+ T cell proliferation and enhance antigen-specific B cell responses.

and adjuvants $(29,31,42,43)$. Therefore, we used this in vitro method to evaluate the capacity of P1 to act as an adjuvant and enhance an antigen-specific humoral response using OVA as a model antigen. Our results clearly indicated that $\mathrm{P} 1$, when in an oligomeric state, enhanced the number of OVA-specific B cells by either favoring antigen presentation and/or T-B interaction. Both OVA-specific B-cell surface IgA and IgG were detected, whereas specific antibody secretion was undetectable at day $5 / 7$, suggesting that in this in vitro model, class switching occurred, but B cells did not develop into plasmablasts. Specific antibody secretion in such in vitro immunization system would require additional stimulations with agents such as CD40L and other cytokines or repeated sensitization with antigen. Nevertheless, the adjuvant function of $\mathrm{P} 1$ should be confirmed in vivo upon intranasal immunization of P1-adjuvanted antigens.

Altogether, our results show that in addition to its protective vaccine antigen properties contributing to full protection against repeated mucosal SHIV infection $(8,12)$ and to inducing in a phase I clinical trial in human mucosal P1-specific IgA with in vitro HIV transcytosis blocking properties $(8,12), \mathrm{P} 1$ acts as an adjuvant, as we now demonstrated here. We show that P1 adjuvant activity occurs in a two-step mode of action during nasal vaccination. In the first step, P1 initiates the very first immune responses at nasal epithelial surface, by producing TSLP, a molecule considered as a strong adjuvant, together with CCL20, CCL2, MMP-9 and IL-10, cytokine determinant in the mucosal immune response. This process is initiated by $\mathrm{P} 1$ interaction with Galactosyl Ceramide, the epithelial receptor for HIV-1 (9-11), activates calcium influx and the PAR-2 and calcineurin pathways, and is regulated by the microRNA miR4485. In the second step, P1 modulates mucosal DCs by inducing the expression of maturation markers, promoting chemokine release that can recruit adaptive immune cells and cytokine secretion that can polarize adaptive immunity into a Th2 and IgA preferential fashion. As a result, $\mathrm{P} 1$-modulated DCs promote CD4+ $\mathrm{T}$ cell proliferation and enhance antigen $\mathrm{B}$ cell-specific responses, as summarized in Figure 7.

Overall, the present study reveals that $\mathrm{P} 1$ is a multi-functional protein with a strong vaccine potential, namely as an immunogen in a fully protective HIV-1 vaccine candidate (8, 12) but also as a promising adjuvant that can combine with other mucosal vaccines.

\section{DATA AVAILABILITY STATEMENT}

The data has been uploaded to NCBI - GSE157449. Other raw data supporting the conclusions of this article will be made available by the authors, without undue reservation, to any qualified researcher. 


\section{AUTHOR CONTRIBUTIONS}

LX, DT, and MB conceived the experiments. LX, DT, and MB performed and analyzed experiments. LX and MB wrote the manuscript. All authors contributed to the article and approved the submitted version.

\section{FUNDING}

Agence Nationale de Recherche sur le SIDA et les Hepatites (AO2015-2-17046). Fondation pour la Recherche Medicale (Grant : « Equipe FRM » EQU201903007830).

\section{REFERENCES}

1. Boyaka PN. Inducing Mucosal IgA: A Challenge for Vaccine Adjuvants and Delivery Systems. J Immunol (2017) 199(1):9-16. doi: 10.4049/ jimmunol.1601775

2. Fujkuyama Y, Tokuhara D, Kataoka K, Gilbert RS, McGhee JR, Yuki Y, et al. Novel vaccine development strategies for inducing mucosal immunity. Expert Rev Vaccines (2012) 11(3):367-79. doi: 10.1586/erv.11.196

3. Brandtzaeg P. Potential of nasopharynx-associated lymphoid tissue for vaccine responses in the airways. Am J Respir Crit Care Med (2011) 183 (12):1595-604. doi: 10.1164/rccm.201011-1783OC

4. Zaman M, Chandrudu S, Toth I. Strategies for intranasal delivery of vaccines. Drug Delivery Transl Res (2013) 3(1):100-9. doi: 10.1007/s13346-012-0085-Z

5. Jabbal-Gill I. Nasal vaccine innovation. J Drug Target (2010) 18(10):771-86. doi: 10.3109/1061186x.2010.523790

6. Lycke N, Lebrero-Fernandez C. ADP-ribosylating enterotoxins as vaccine adjuvants. Curr Opin Pharmacol (2018) 41:42-51. doi: 10.1016/j.coph.2018. 03.015

7. Johansson EL, Wassen L, Holmgren J, Jertborn M, Rudin A. Nasal and vaginal vaccinations have differential effects on antibody responses in vaginal and cervical secretions in humans. Infect Immun (2001) 69(12):7481-6. doi: 10.1128/iai.69.12.7481-7486.2001

8. Bomsel M, Tudor D, Drillet AS, Alfsen A, Ganor Y, Roger MG, et al. Immunization with HIV-1 gp41 subunit virosomes induces mucosal antibodies protecting nonhuman primates against vaginal SHIV challenges. Immunity (2011) 34(2):269-80. doi: 10.1016/j.immuni.2011.01.015

9. Alfsen A, Bomsel M. HIV-1 gp41 envelope residues 650-685 exposed on native virus act as a lectin to bind epithelial cell galactosyl ceramide. $J$ Biol Chem (2002) 277(28):25649-59. doi: 10.1074/jbc.M200554200

10. Bomsel M. Transcytosis of infectious human immunodeficiency virus across a tight human epithelial cell line barrier. Nat Med (1997) 342-7(1):42-7. doi: 10.1038/nm0197-42

11. Magérus-Chatinet A, Yu H, Garcia S, Ducloux E, Terris B, Bomsel M. Galactosyl ceramide expressed on dendritic cells can mediate HIV-1 transfer from monocyte derived dendritic cells to autologous $\mathrm{T}$ cells. Virology (2007) 362(1):67-74. doi: 10.1016/j.virol.2006.11.035

12. Leroux-Roels G, Maes C, Clement F, van Engelenburg F, van den Dobbelsteen M, Adler M, et al. Randomized Phase I: Safety, Immunogenicity and Mucosal Antiviral Activity in Young Healthy Women Vaccinated with HIV-1 Gp41 P1 Peptide on Virosomes. PLoS One (2013) 8(2):e55438. doi: 10.1371/ journal.pone.0055438

13. Takai T. TSLP Expression: Cellular Sources, Triggers, and Regulatory Mechanisms. Allergol Int (2012) 61(1):3-17. doi: 10.2332/allergolint.11RAI-0395

14. Ito T, Liu Y-J, Arima K. Cellular and Molecular Mechanisms of TSLP Function in Human Allergic Disorders - TSLP Programs the "Th2 code" in Dendritic Cells. Allergol Int (2012) 61(1):35-43. doi: 10.2332/allergolint.11RAI-0376

15. Fontenot D, He H, Hanabuchi S, Nehete PN, Zhang M, Chang M, et al. TSLP production by epithelial cells exposed to immunodeficiency virus triggers DCmediated mucosal infection of CD4+ T cells. Proc Natl Acad Sci USA (2009) 106(39):16776-81. doi: 10.1073/pnas.0907347106

\section{ACKNOWLEDGMENTS}

The authors wish to thank Clotilde Ramandriapita, Cochin Institute, for helpful discussion about calcium measurements and Thomas Guilbert from IMAGIC platform at Cochin Institute for calcium measurement experiments and their analyses. This study was supported by grants from l'Agence Nationale de Recherches sur le Sida et les Hépatites Virales (ANRS) (AO2015-2-17046) and from the Fondation pour la Recherche Medicale (Grant : «Equipe FRM ») to MB. LX was supported by the China Scholarship Council.

16. Zhou Z, Xu L, Sennepin A, Federici C, Ganor Y, Tudor D, et al. The HIV-1 viral synapse signals human foreskin keratinocytes to secrete thymic stromal lymphopoietin facilitating HIV-1 foreskin entry. Mucosal Immunol (2018) 11 (1):158-71. doi: 10.1038/mi.2017.23

17. Van Roey GA, Arias MA, Tregoning JS, Rowe G, Shattock RJ. Thymic stromal lymphopoietin (TSLP) acts as a potent mucosal adjuvant for HIV-1 gp140 vaccination in mice. Eur J Immunol (2012) 42(2):353-63. doi: 10.1002/ eji.201141787

18. Hatayama T, Segawa R, Mizuno N, Eguchi S, Akamatsu H, Fukuda M, et al. All-Trans Retinoic Acid Enhances Antibody Production by Inducing the Expression of Thymic Stromal Lymphopoietin Protein. J Immunol (2018) 200 (8):2670-6. doi: 10.4049/jimmunol.1701276

19. Joo S, Fukuyama Y, Park EJ, Yuki Y, Kurashima Y, Ouchida R, et al. Critical role of TSLP-responsive mucosal dendritic cells in the induction of nasal antigen-specific IgA response. Mucosal Immunol (2017) 10(4):901-11. doi: $10.1038 / \mathrm{mi} .2016 .103$

20. Sallusto F, Lanzavecchia A. Efficient presentation of soluble antigen by cultured human dendritic cells is maintained by granulocyte/macrophage colony-stimulating factor plus interleukin 4 and downregulated by tumor necrosis factor alpha. J Exp Med (1994) 179(4):1109-18. doi: 10.1084/ jem.179.4.1109

21. Dong H, Hu Y, Liu L, Zou M, Huang C, Luo L, et al. Distinct roles of short and long thymic stromal lymphopoietin isoforms in house dust mite-induced asthmatic airway epithelial barrier disruption. Sci Rep (2016) 6:39559. doi: $10.1038 /$ srep39559

22. Bjerkan L, Schreurs O, Engen SA, Jahnsen FL, Baekkevold ES, Blix IJ, et al. The short form of TSLP is constitutively translated in human keratinocytes and has characteristics of an antimicrobial peptide. Mucosal Immunol (2015) 8 (1):49-56. doi: 10.1038/mi.2014.41

23. Schmittgen TD, Livak KJ. Analyzing real-time PCR data by the comparative C (T) method. Nat Protoc (2008) 3(6):1101-8. doi: 10.1038/nprot.2008.73

24. Wygrecka M, Dahal BK, Kosanovic D, Petersen F, Taborski B, von Gerlach S, et al. Mast cells and fibroblasts work in concert to aggravate pulmonary fibrosis: role of transmembrane SCF and the PAR-2/PKC-alpha/Raf-1/p44/42 signaling pathway. Am J Pathol (2013) 182(6):2094-108. doi: 10.1016/ j.ajpath.2013.02.013

25. Kelso EB, Lockhart JC, Hembrough T, Dunning L, Plevin R, Hollenberg MD, et al. Therapeutic promise of proteinase-activated receptor-2 antagonism in joint inflammation. J Pharmacol Exp Ther (2006) 316(3):1017-24. doi: 10.1124/jpet.105.093807

26. Conche C, Boulla G, Trautmann A, Randriamampita C. T cell adhesion primes antigen receptor-induced calcium responses through a transient rise in adenosine 3',5'-cyclic monophosphate. Immunity (2009) 30(1):33-43. doi: 10.1016/j.immuni.2008.10.020

27. Guichard V, Bonilla N, Durand A, Audemard-Verger A, Guilbert T, Martin B, et al. Calcium-mediated shaping of naive CD4 T-cell phenotype and function. Elife (2017) 6:1-26. doi: 10.7554/eLife.27215

28. Duchemin M, Khamassi M, Xu L, Tudor D, Bomsel M. IgA Targeting Human Immunodeficiency Virus-1 Envelope gp41 Triggers Antibody-Dependent Cellular Cytotoxicity Cross-Clade and Cooperates with gp41-Specific IgG to Increase Cell Lysis. Front Immunol (2018) 9(244):1-30. doi: 10.3389/ fimmu.2018.00244 
29. Yeh CY, Yeh TH, Jung CJ, Chen PL, Lien HT, Chia JS. Activated human nasal epithelial cells modulate specific antibody response against bacterial or viral antigens. PloS One (2013) 8(2):e55472. doi: 10.1371/journal.pone.0055472

30. Qin T, Yin Y, Wang X, Liu H, Lin J, Yu Q, et al. Whole inactivated avian Influenza H9N2 viruses induce nasal submucosal dendritic cells to sample luminal viruses via transepithelial dendrites and trigger subsequent DC maturation. Vaccine (2015) 33(11):1382-92. doi: 10.1016/j.vaccine.2015.01.022

31. Jung YS, Matsumoto SE, Yamashita M, Tomimatsu K, Teruya K, Katakura Y, et al. Propionibacterium acnes acts as an adjuvant in in vitro immunization of human peripheral blood mononuclear cells. Biosci Biotechnol Biochem (2007) 71(8):1963-9. doi: 10.1271/bbb.70159

32. Tsilingiri K, Fornasa G, Rescigno M. Thymic Stromal Lymphopoietin: To Cut a Long Story Short. Cell Mol Gastroenterol Hepatol (2017) 3(2):174-82. doi: 10.1016/j.jcmgh.2017.01.005

33. Biton M, Levin A, Slyper M, Alkalay I, Horwitz E, Mor H, et al. Epithelial microRNAs regulate gut mucosal immunity via epithelium-T cell crosstalk. Nat Immunol (2011) 12(3):239-46. doi: 10.1038/ni.1994

34. Sripada L, Singh K, Lipatova AV, Singh A, Prajapati P, Tomar D, et al. hsamiR-4485 regulates mitochondrial functions and inhibits the tumorigenicity of breast cancer cells. J Mol Med (Berl) (2017) 95(6):641-51. doi: 10.1007/ s00109-017-1517-5

35. Coughlin SR. Thrombin signalling and protease-activated receptors. Nature (2000) 407:258. doi: 10.1038/35025229

36. Wilson SR, The L, Batia LM, Beattie K, Katibah GE, McClain SP, et al. The epithelial cell-derived atopic dermatitis cytokine TSLP activates neurons to induce itch. Cell (2013) 155(2):285-95. doi: 10.1016/j.cell.2013.08.057

37. Hogan PG, Chen L, Nardone J, Rao A. Transcriptional regulation by calcium, calcineurin, and NFAT. Genes Dev (2003) 17(18):2205-32. doi: 10.1101/ gad. 1102703

38. Coffman RL, Sher A, Seder RA. Vaccine adjuvants: putting innate immunity to work. Immunity (2010) 33(4):492-503. doi: 10.1016/j.immuni.2010.10.002

39. Schon-Hegrad MA, Oliver J, McMenamin PG, Holt PG. Studies on the density, distribution, and surface phenotype of intraepithelial class II major histocompatibility complex antigen (Ia)-bearing dendritic cells (DC) in the conducting airways. J Exp Med (1991) 173(6):1345-56. doi: 10.1084/ jem.173.6.1345

40. Brandtzaeg P. Mucosal immunity: induction, dissemination, and effector functions. Scand J Immunol (2009) 70(6):505-15. doi: 10.1111/j.13653083.2009.02319.x

41. Rimoldi M, Chieppa M, Salucci V, Avogadri F, Sonzogni A, Sampietro GM, et al. Intestinal immune homeostasis is regulated by the crosstalk between epithelial cells and dendritic cells. Nat Immunol (2005) 6(5):507-14. doi: 10.1038/ni1192

42. Borrebaeck CA, Danielsson L, Moller SA. Human monoclonal antibodies produced from L-leucine methyl ester-treated and in vitro immunized peripheral blood lymphocytes. Biochem Biophys Res Commun (1987) 148 (3):941-6. doi: 10.1016/S0006-291X(87)80223-1

43. Wijkhuisen A, Savatier A, Cordeiro N, Leonetti M. Production of antigenspecific human IgGs by in vitro immunization. BMC Biotechnol (2016) 16:22. doi: 10.1186/s12896-016-0253-1

44. Kurt-Jones EA, Popova L, Kwinn L, Haynes LM, Jones LP, Tripp RA, et al. Pattern recognition receptors TLR4 and CD14 mediate response to respiratory syncytial virus. Nat Immunol (2000) 1(5):398-401. doi: 10.1038/80833
45. Nazli A, Kafka JK, Ferreira VH, Anipindi V, Mueller K, Osborne BJ, et al. HIV-1 gp120 induces TLR2- and TLR4-mediated innate immune activation in human female genital epithelium. J Immunol (2013) 191(8):4246-58. doi: 10.4049/jimmunol.1301482

46. George JA, Kim SB, Choi JY, Patil AM, Hossain FMA, Uyangaa E, et al. TLR2/ MyD88 pathway-dependent regulation of dendritic cells by dengue virus promotes antibody-dependent enhancement via Th2-biased immunity. Oncotarget (2017) 8(62):106050-70. doi: 10.18632/oncotarget.22525

47. Saghazadeh A, Rezaei N. Implications of Toll-like receptors in Ebola infection. Expert Opin Ther Targets (2017) 21(4):415-25. doi: 10.1080/14728222.2017.1299128

48. Wang Q, Du J, Zhu J, Yang X, Zhou B. Thymic stromal lymphopoietin signaling in CD4(+) $\mathrm{T}$ cells is required for TH2 memory. J Allergy Clin Immunol (2015) 135(3):781-91.e3. doi: 10.1016/j.jaci.2014.09.015

49. Kamekura R, Kojima T, Koizumi J, Ogasawara N, Kurose M, Go M, et al. Thymic stromal lymphopoietin enhances tight-junction barrier function of human nasal epithelial cells. Cell Tissue Res (2009) 338(2):283-93. doi: 10.1007/s00441-009-0855-1

50. Vliagoftis H, Schwingshackl A, Milne CD, Duszyk M, Hollenberg MD, Wallace JL, et al. Proteinase-activated receptor-2-mediated matrix metalloproteinase-9 release from airway epithelial cells. J Allergy Clin Immunol (2000) 106(3):537-45. doi: 10.1067/mai.2000.109058

51. Sun JC, Ugolini S, Vivier E. Immunological memory within the innate immune system. EMBO J (2014) 33(12):1295-303. doi: 10.1002/ embj.201387651

52. Takano K, Kojima T, Go M, Murata M, Ichimiya S, Himi T, et al. HLA-DR- and CD11c-positive dendritic cells penetrate beyond well-developed epithelial tight junctions in human nasal mucosa of allergic rhinitis. $J$ Histochem Cytochem (2005) 53(5):611-9. doi: 10.1369/jhc.4A6539.2005

53. Ziegler SF. Thymic stromal lymphopoietin (TSLP) and allergic disease. J Allergy Clin Immunol (2012) 130(4):845-52. doi: 10.1016/j.jaci.2012.07.010

54. Meng H, Li H, Ohe R, Naing YA, Yang S, Kabasawa T, et al. Thymic stromal lymphopoietin in tonsillar follicular dendritic cells correlates with elevated serum immunoglobulin A titer by promoting tonsillar immunoglobulin A class switching in immunoglobulin A nephropathy. Transl Res (2016) 176:117. doi: $10.1016 /$ j.trsl.2016.04.008

55. Smelter D, Thompson M, Meuchel L, Townsend E, Ryu A, Pabelick C, et al. Thymic Stromal Lymphopoietin (TSLP) and Airway Smooth Muscle. FASEB J (2009) 23(1_supplement):622.6-6. doi: 10.1096/fasebj.23.1_supplement.622.6

56. Anjuere F, George-Chandy A, Audant F, Rousseau D, Holmgren J, Czerkinsky C. Transcutaneous immunization with cholera toxin $\mathrm{B}$ subunit adjuvant suppresses IgE antibody responses via selective induction of Th1 immune responses. J Immunol (2003) 170(3):1586-92. doi: 10.4049/jimmunol.170.3.1586

Conflict of Interest: The authors declare that the research was conducted in the absence of any commercial or financial relationships that could be construed as a potential conflict of interest.

Copyright (C) $2021 \mathrm{Xu}$, Tudor and Bomsel. This is an open-access article distributed under the terms of the Creative Commons Attribution License (CC BY). The use, distribution or reproduction in other forums is permitted, provided the original author(s) and the copyright owner(s) are credited and that the original publication in this journal is cited, in accordance with accepted academic practice. No use, distribution or reproduction is permitted which does not comply with these terms. 